\title{
Aortic Aneurysm
}

National Cancer Institute

\section{Source}

National Cancer Institute. Aortic Aneurysm. NCI Thesaurus. Code C26697.

A sac formation resulting from the localized dilatation of the wall of the aorta. 